\title{
The Use of Comaps to Explore the Spatial and Temporal Dynamics of Fire Incidents: A Case Study in South Wales, United Kingdom*
}

\author{
Jonathan Corcoran \\ University of Queensland \\ Gary Higgs \\ University of Glamorgan
}

Chris Brunsdon

University of Leicester

\author{
Andrew Ware \\ University of Glamorgan
}

This article reports on the results from a spatiotemporal analysis of disaggregate fire incident data. The innovative analysis presented here focuses on the exploration of spatial and temporal patterns for four principal fire incident categories: property, vehicle, secondary fires, and malicious false alarms. This research extends previous work on spatial exploration of spatiotemporal patterns by demonstrating the benefits of comaps and kernel density estimation in examining temporal and spatiotemporal dynamics in calls for services. Results indicate that fire incidents are not static in either time or space and that spatiotemporal variation is related to incident type. The application of these techniques has the potential to inform policy decisions both from a reactive, resourceallocation perspective and from a more proactive perspective, such as through spatial targeting of preventive measures. Key Words: comap, fire, GIS, KDE, risk surface, spatiotemporal analysis.

$\mathbf{F}$ ire, whether malicious or unintentional, has either directly or indirectly affected the majority of the populace, imposing significant economic costs, psychological damage, physical injuries, and death. The economic cost of fire is far from trivial; the Office of the Deputy Prime Minister estimated the cost in 2003 to be $£ 7.7 \mathrm{bn}$ $(\sim \$ 14.3 \mathrm{bn})$ in England and Wales, with each domestic fire on average costing $£ 25,000$ ( \$46,400) (ODPM 2005b). In addition, hoax calls contribute a large and unnecessary cost to the fire service-estimated for the Welsh fire services alone at $£ 700,000(\sim \$ 1.3 \mathrm{~m})$ in 2005 (BBC News 2006). For the individuals and families involved there are of course more personal aspects related to the loss of life, injuries sustained through burns and smoke inhalation, and the psychological effects that such incidents may cause. In the United Kingdom, injuries caused by fire and flame are comparatively more serious than other home-related injuries (DiGuiseppi et al. 2000). In 2004 there were 405 deaths (0.68 per 100,000 population) and 12,200 (21.4 per 100,000 population) injuries recorded in the United Kingdom resulting from dwelling fires (ODPM 2005a). This compares to a fire facility rate of 0.1 per 100,000 population in Australia and 0.07 in New Zealand (2003-2004 figures; Australasian Fire Authorities Council 2005). Figures provided in Lapidus et al. (1998) suggest that in the United States an estimated 5,000 (1.84 per 100,000 population) people die each year from residential fires.

\footnotetext{
${ }^{*}$ We would like to thank the South Wales Fire and Rescue Service for access to the data on which the article is based. In particular, we thank Andy Marles and Dave Bennett of the South Wales Fire and Rescue Service for their help and advice on various aspects of the data sets. The interpretations of the analysis, however, are solely those of the authors and do not necessarily reflect the views and opinions of the Service or any of its employees. The work uses 2001 Census and GIS boundary data obtained via MIMAS CASWEB and EDINA UKBORDERS; academic services both supported by ESRC and JISC. These data are copyright of the Crown and are reproduced with permission of the Controller of HMSO.
} 
In contrast to the examination of crime patterns, the geographical variations of fire incidence have been the subject of relatively less attention. The contention of this article is that geographical studies of fire incidence represent an important public health issue in which geographical information systems (GIS) have a potentially significant role to play in terms of risk identification, resource targeting and routing of fire personnel and equipment, allocation of preventative measures (such as smoke alarms), and policy evaluation. Although there has been a relatively large published literature on GIS-based techniques for the identification, assessment, and management of wildfires (see, e.g., Pew and Larsen 2001; Jaiswal et al. 2002; Vakalis et al. 2004a, 2004b; Hernandez-Leal, Arbelo, and Gonzalez-Calvo 2006), there has been much less research on spatiotemporal patterns of property fires (PFs) and vehicle fires (VFs) and of hoax calls. Among the emergency services such as the police, fire, and ambulance services, there is an increasing recognition of the potential benefits of using GIS particularly as related to mapping applications. GIS has been used extensively, for example, to assist in vehicle dispatch and the identification of optimal fire station locations (Liu, Huang, and Chandramouli 2006). In addition, GIS are increasingly being used in the preparation of integrated risk management plans (IRMPs), which have been required of each fire service in the United Kingdom since 2004 (ODPM 2005b). These plans are strategy documents that outline how each fire service will address its prevention and response objectives. Ormsby (2005) provides some recent examples of the wider use of GIS within the fire service in terms of prevention through targeting buildings for inspection and in examining spatial patterns of buildings at risk. Increasingly, GIS is being used to visualize maps of fire safety risk assessments and to organize detailed programs of inspections or educational intervention campaigns within a forces' area. Some fire and rescue services are also using GIS in fire safety checks by targeting hotspots of potentially high risk based on socioeconomic characteristics, reinforced by analyses of previous patterns of fire incidence. However, many of these studies are context-dependent and more research is needed to examine how transferable these findings are to other countries.
Substantial interest in GIS-based analyses of crime data has resulted in a number of dedicated texts (e.g., Weisburd and McEwen 1997; Harries 1999; Goldsmith et al. 2000; Hirschfield and Bowers 2001; Leipnik and Albert 2003; Paulsen, Robinson, and Robinson 2004; Boba, 2005; Chainey and Ratcliffe 2005; Eck et al. 2005), but relatively few studies describe the application of GIS techniques in exploring spatial and temporal dynamics of fire incidents (Merrall 2001). There are parallels to the types of analyses conducted in crime applications and in particular to issues surrounding techniques used to explore detailed patterns of disaggregate point level data, however more studies are needed to fill the gaps in this literature. Some research has analyzed the socioeconomic characteristics of areas in relation to the levels of fire incidences experienced. Jennings (1999) provides the most recent review of studies examining relationships between socioeconomic factors and fire incidence and risk. That work, which drew on studies in the United States largely from an urban (residential fires) perspective, includes a review of factors such as abandonment and property decline on intraurban variations in fire rates. Jennings also drew on an earlier study of three U.K. urban areas that found that factors such as the age of housing, housing tenure, and socioeconomic status (social class, unemployment status, ethnicity) are correlated with fire rates (Chandler, Chapman, and Hallington 1984). A more recent GIS-based socioeconomic study of fire incidents (Corcoran et al. 2007) investigated four types of fire incidents and their association with thirty-two census-derived variables for South Wales, United Kingdom. Results indicate that wards in which residents have relatively lower levels of educational attainment are associated with higher proportions of $\mathrm{VFs}, \mathrm{PFs}$, and secondary fires (SFs), and wards with lower proportions of white residents are associated with more PFs. In addition, wards that exhibit a higher level of crowding are more susceptible to VFs and SFs. Our previous research (Corcoran et al. 2007) suggests that malicious false alarms (FAs) are more prevalent in areas that have lower proportions of childless couples and lower proportions of car owners. The research presented in this article extends the spatial exploration of patterns identified in our previous work by demonstrating the benefits of comaps and 
kernel density estimation (KDE) in examining temporal and spatiotemporal dynamics in calls for services. These techniques in themselves are not innovative, but to our knowledge they have not been applied to calls for service data, and their potential in examining spatiotemporal patterns in fire incidence remains underresearched. Specifically, we explore the spatial, temporal, and spatiotemporal dynamics for four fire incident types (PFs, VFs, SFs, and FAs) for a fire service area in South Wales, United Kingdom, in order to illustrate the potential of such techniques and discuss their transferability to other contexts.

The rest of the article is structured in four sections. We first introduce the study area and the data sets used, then focus on the techniques used to examine spatial and temporal patterns.
The next section presents the results from the analysis, and the following section discusses the findings coupled with the advantages and limitations of the approach we have taken and ways in which this research can be progressed. The final section draws on the findings from this research and offers some preliminary conclusions.

\section{Methodology}

Study Area and Data Sources/Preparation

In this study we have access to detailed disaggregate data in the form of a database of calls for service to fire incidents for a four-year period (1 January 2001-31 December 2004) across the South Wales Fire Rescue Service (SWFRS) command region (Figure 1). Fifty fire stations

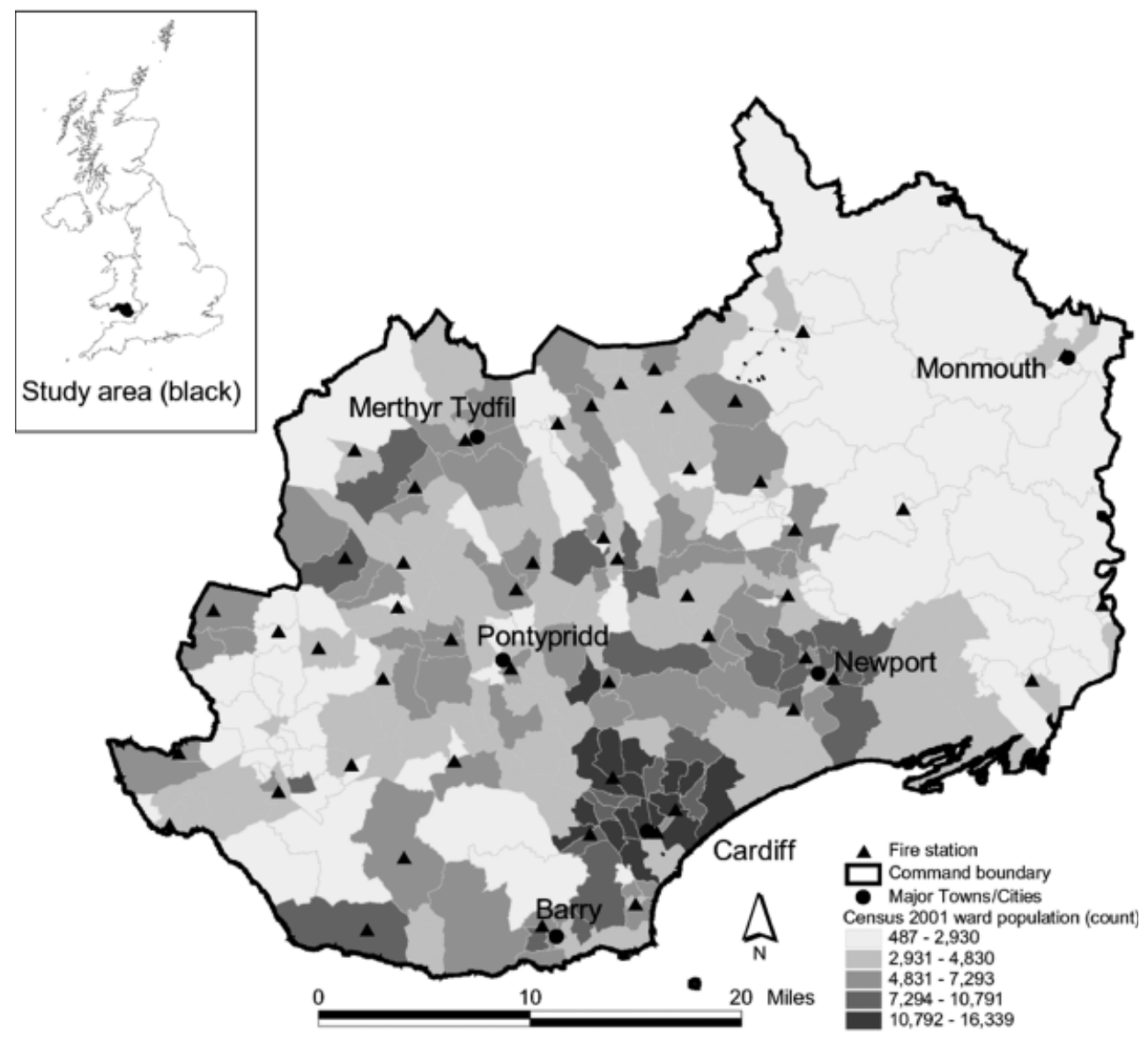

Figure 1 South Wales Fire and Rescue Service command region. 
Table 1 Descriptions of fire incident types

\begin{tabular}{llcc}
\hline Code & \multicolumn{1}{c}{ Definition } & $\begin{array}{c}\text { Number of } \\
\text { incidents }\end{array}$ & $\begin{array}{c}\text { Percentage } \\
\text { of total } \\
\text { incidents }\end{array}$ \\
\hline PF & $\begin{array}{l}\text { Property fires: All fires involving property (e.g., dwellings, public buildings, work- } \\
\text { places). }\end{array}$ & 13,157 & 13.39 \\
VF & $\begin{array}{l}\text { Vehicle fires: Vehicles (except derelict or abandoned vehicles). } \\
\text { SF }\end{array}$ & $\begin{array}{l}\text { Secondary fires: Derelict buildings/vehicles; refuse or refuse containers; outdoor } \\
\text { structures (e.g., fence, gate, road sign); grass. }\end{array}$ & 16,723 \\
FA & False alarms: False alarms deemed malicious/deliberate. & 62,444 & 17.02 \\
\hline
\end{tabular}

provide cover for a population of just fewer than 1.4 million, which includes six major urban centers: Cardiff, Merthyr Tydfil, Newport, Pontypridd, Monmouth, and Barry. Each call for service comprises a date, a time, a grid reference, and the type of incident. A description of fire incident types is provided in Table 1 , which distinguishes the main categories of fires. Calls for fire service data were used, as the time stamp recorded is likely to be relatively close to the actual time of the ignition of the fire. Though this may not be the case in some instances of smoldering fires or fires in remote regions (e.g., grassland fires), such exceptions were deemed to not significantly affect the subsequent analysis because the overwhelming majority of the recorded incidents occurred in populated regions. In all, data were collected for 13,286 primary fires involving property (PFs), 16,872 VFs, $62,895 \mathrm{SFs}$, and 6,112 FAs, amounting to just over 99,000 incidents in total. Data not containing any spatial reference totaled $685(0.69$ percent), including $94 \mathrm{PFs}$ (0.7 percent), 106 VFs (0.62 percent), 328 SFs ( 0.52 percent), and 157 FAs (2.57 percent), and these were omitted from the analysis.

An important consideration when performing a spatial analysis on incident data is the accuracy of the spatial location assigned to each incident. Since 1999 the SWFRS has assigned a spatial reference to all incidents recorded; however, over time there have been refinements both to the number of records contained in the geocoding gazetteer and through the introduction of hand-held global positioning (GPS) units. Between 1999 and 2002 the gazetteer used to spatially reference each incident contained a total of 30,000 location records, however there are cases where incidents could be tagged with "parish references" that equated to a single location within a fire service defined local area. Using such a spatial granularity un- doubtedly introduces some spatial errors where the mapped location of an incident may not equate to the exact location of a fire. For incidents occurring post-2002 the geocoding gazetteer was extended to include more than 600,000 location records. Some spatial errors are still possible when, as a result of information provided by the caller, fire resources are mobilized to street references and in some cases parish references as opposed to exact locations. In the case of nonaddressable locations (such as stretches of roads), incidents are often associated with the location of the nearest roundabout or intersection.

An additional important improvement that potentially impacts on the accuracy of the geocoding was the introduction of handheld GPS units in 2002. These units provide a grid reference of the fire that is radioed back to headquarters via a verbal radio message. This technology is likely to have the greatest influence for nonproperty fires (e.g., grassland fires and VFs) where an exact reference in the gazetteer is less likely. Overall, changes in the SWFS geocoding procedures over the time period under investigation here could have influenced patterns in some incident types but such analysis was beyond the scope of this preliminary exploratory study and will form the basis of our future studies in this area.

Using the geographical locations, all of the incidents per ward were aggregated for each incident category using ArcGIS 9.1 (2005). The incidence rates (per 1,000 population) were then calculated using the population of the tract at the time of the 2001 Census of Population. Using resident population rather than the number of households as the denominator is common with other studies and was justified in this study by the strong correlation (not presented here) between the number of households and the number of people residing in wards in 
Table 2 Change in incident rates by type over the period 2001 to 2004 (rate per 1,000 population)

\begin{tabular}{lllllc}
\hline \multicolumn{1}{c}{ Code } & $\mathbf{2 0 0 1}$ & $\mathbf{2 0 0 2}$ & $\mathbf{2 0 0 3}$ & $\mathbf{2 0 0 4}$ & Overall change \\
\hline Property fires & 2.15 & 1.89 & 1.83 & 1.64 & $-24 \%$ \\
Vehicle fires & 2.46 & 2.48 & 2.63 & 2.10 & $-15 \%$ \\
Secondary fires & 9.98 & 8.20 & 11.91 & 7.32 & $-27 \%$ \\
False alarms & 1.03 & 0.84 & 0.77 & 0.72 & $-30 \%$ \\
\hline
\end{tabular}

England and Wales. Due to the size of the population in the study area $(\sim 1.4$ million in the 2001 Census) and in common with GIS-based crime studies at this geographical scale, rates are calculated per 1,000 population.

Analyzing the change of incident rate (Table 2) highlights the fact that for each incident type there has been a reduction over the four-year period. The most marked reduction in rate is for FA incidents, followed by SFs, then PFs, and finally VFs. For SFs there was a marked increase (45 percent) in rate during 2003 that subsequently reduced the following year by 39 percent that equated to an overall 27 percent decrease in the rate over the four-year period. Inspection of the time series highlights that the increase experienced during 2003 was an accentuation of the same temporal patterns in other years (see the discussion of temporal dynamics in the Results section), however no further data were available to help explain this increase.

\section{Techniques Used}

We used three kinds of techniques in this study: temporal, spatial, and spatiotemporal. To investigate temporal patterns, simple line and circular plots were used for different granularities of time (i.e., hourly, daily, and monthly). Spatial techniques first explored the concentration through the use of a cumulative sum technique based on wards. This was coupled with the results from a kernel density "risk surface" from our previous research (Corcoran et al. 2007) to highlight the spatial variability across the study area and to show how these variations changed by incident category. In this article we explore the interaction of space and time using a technique called the comap (Brunsdon 2001). The advantage of comaps in this context is that they are able to illustrate the entire time period under study in a single visualization, an advantage over the more traditional map animation methods used to explore spatiotemporal dynamics. Es- pecially where there are numerous changes in pattern, a synoptic visualization approach such as the comap offers the potential for greater insight to be achieved.

The comap builds on a technique known as the co-plot (Cleveland 1993) that is used to examine the relationship between a pair of variables (in our case the $x$ and $y$ locations of a particular fire incident) that are conditioned using a third variable, $z$ (in our case a measure of time, such as hour of day). Using this method it is possible to investigate the relationship between the locations of fire incidents ( $x$ and $y$ ) and their variation given different values of time $z$. For the purposes of our investigation we explored the effect of different granularities of time $z$ (i.e., month, day, and hour) and their effect on the spatial distribution of fire incidents $(x$ and $y)$. This process was repeated for each incident type. The comap works by subsetting observations, in this case individual fire incidents (on the basis of the conditioning variable $z$ ), and represents the output as a scatter plot. This is repeated for each subset, and the individual plots are arranged in an ordered set of panels such that the relationship of $x$ and $y$ can be explored as $z$ increases. The subsetting principles are governed by two main rules: first, the range of each subset must have some overlap with each adjoining subset, and second, each subset must contain approximately the same number of observations. The main reason for these rules is that the resulting output should not be an artifact of the classification process; for example, deciding to subset the data into hourly sets may occlude a pattern that is not readily discernable using an hourly classification.

The distinction between the co-plot and comap is simply that if the data used are geographical in nature (i.e., they possess $x, y$ coordinates), then each panel in effect becomes an individual map-hence the term comap (Brunsdon 2001). Interpretation of the panels in relation to the ranges is given in Figure 2. 
A
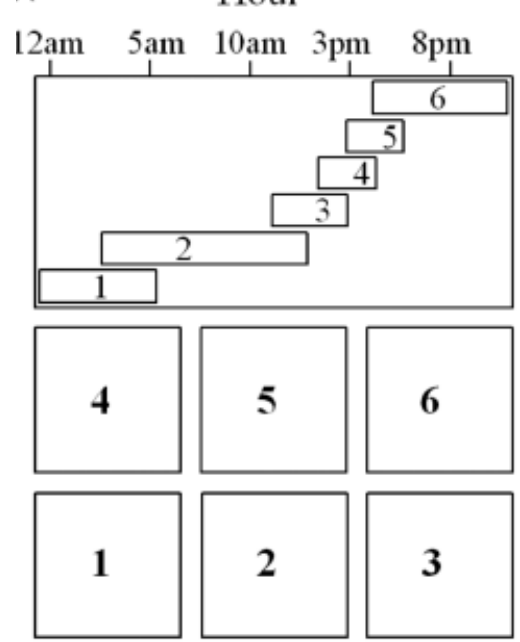

B

Hour

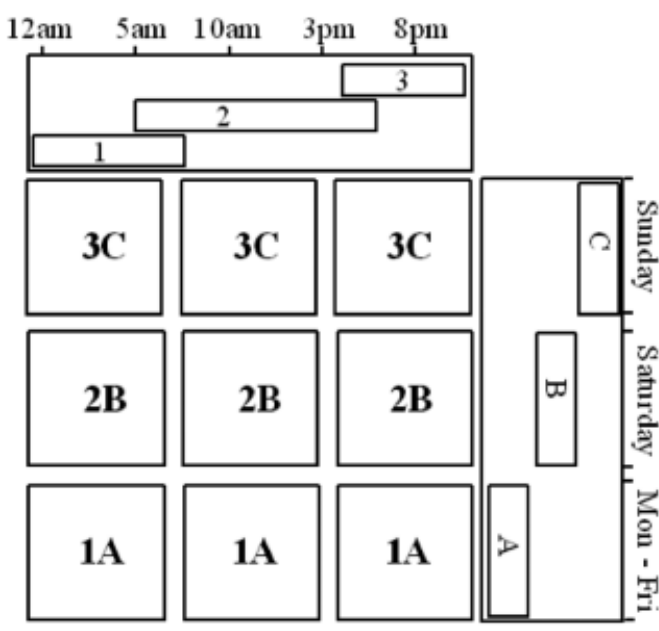

Figure 2 (A) Interpretation of a univariate comap. (B) Interpretation of a bivariate comap.

Figure 2A shows six panels conditioned using the variable of hour (numbered 1 to 6). Each panel corresponds to a range of time, with the lower ranges appearing first. Figure 2B shows the interpretation of a bivariate comap, where the data are conditioned on two variables (hour as before, in addition to day). Note that in the bivariate comap the ranges for the second conditioning variable do not overlap as they do with hour. This is because the day period is a categorical variable and not continuous as hour and therefore must be treated differently as it is no longer possible to select subsets with the same numbers of observations. In this case days are grouped together in a manner that attempts to achieve a similar number of observations per panel. This situation is also the same as that for monthly variations and hence is treated in the same way described here. In spite of this modification, the outputs still have potential to offer some useful insights into spatiotemporal patterns.

The use of points in each panel (in the form of a scatter plot) to represent the locations of a particular fire incident can be effective in situations where the number of incidents is limited and/or reasonably well dispersed. Where incidents are both numerous and clustered, the point representation can become difficult to interpret as the actual number of incidents can be impossible to determine. This is especially the case when a single locality is subject to multiple incidents. An improved method of visualization is to apply KDE (Silverman 1986) to create a risk surface. In the case of the comap, a risk surface is produced for each panel, with the darker shading indicating a higher number of fire incidents.

$\mathrm{KDE}$ has become a popular visualization method in situations where the volume of incidents is relatively large and spatially clustered (see, e.g., Brunsdon, Corcoran, and Higgs 2007). The risk surface output of the KDE readily permits identification of areas exhibiting high concentrations of fire incidents in contrast to those showing lower incidence levels. The derivation of the density estimates in this study were based on the physical area of the kernel, however it is recognized that this could be modified to use the underlying population as the denominator. Although use of the underlying population for PFs might generate some interesting results, its use with SFs and VFs seems less applicable, therefore physical area was used as the denominator across all incident types. However, it is acknowledged that the use of alternative denominators could form the basis for future research efforts.

The use of comaps to explore spatiotemporal patterns offers a number of advantages and limitations. Advantages include the fact that the comap is synoptic, in other words the technique 
is capable of representing the entire time period under study in a single visualization. This permits superior comparative analysis to be made between the temporal and spatial dimensions for the whole temporal coverage, in contrast to map animation where each time period is represented as a single visualization. The comap is particularly advantageous over map animation where there are large spatial variations between subsequent time slices (Brunsdon, Corcoran, and Higgs 2007). A second advantage is related to the way the comap technique portions the data into each map panel using Tufte's "small multiple" principle (also known as "small multiple design" or "small multiples"; Tufte 1990, 67). This classification process (i.e., how the data are divided into various temporal divisions) attempts to achieve an overlap between class boundaries in order to achieve a similar amount of data in each map panel and thus avoid the output being a function of the classification process.

A limitation of the comap technique is that it requires both a certain level of familiarity with the underlying concept of the comap creation and the experience needed to interpret the findings from such analysis. This aspect is presented in the next section.

\section{Results}

\section{Spatial Analysis of Fire Incidents}

The first part of the investigation targeted a ward level of analysis (a ward is a geographical unit comparable to a census tract in the United States). Specifically, the total count for each incident type per ward was used to derive a measure of spatial concentration using a cumulative sum method. This permitted examination of the spatial concentration relative to both the study region and the population at risk. The geographical pattern of hoax calls (FA) showed the highest spatial concentration with 13.5 percent of the wards (containing 23.3 percent of the total population) being subject to 50 percent of FA incidents within the study area. This compares to 17.6 percent of the wards and the 25.7 percent of the population therein, accounting for 50 percent of SF incidents, and 18 percent of the wards, with 29 percent of the population accounting for 50 percent of VF incidents. The least spatial concentration is for PF incidents where 21.8 percent of wards (containing 37 percent of the total population) are the subject of 50 percent of all PF incidents.

Often the use of administrative boundaries can place artificial constraints on data and thus influence the interpretation of the results. Therefore the results presented above are supplemented with the findings of a $\mathrm{KDE}$ analysis presented in a previous paper (Corcoran et al. 2007). These results reinforce the spatial variability of fire incidents across the study region, highlighting four key observations:

- The lowest fire incident intensity for all categories is at the eastern side of the region.

- All incident types exhibit a common high spatial concentration in the south of the command region-the consequence of a higher concentration of population in that area (Cardiff, Barry, and Newport).

- FAs show the smallest geographical area affected, with the main concentration around the Cardiff region.

- SFs shows the greatest geographical area affected. Of particular note is the area to the northwest of Pontypridd, which does not exhibit that level of intensity for the other incident types.

\section{Temporal Dynamics}

To explore the temporal dynamics of each incident, simple plots were created for various temporal granularities: monthly, daily, and hourly. For each plot the mean monthly, daily, and hourly values were first computed to derive a percentage deviation from the mean. The resulting output permits a useful way by which temporal rhythms at various granularities can be examined.

Monthly Monthly variations (Figure 3) are most marked in the SF incident type, where there are deviations from the mean during March (+69.59 percent) and April (+97.03 percent), the fewest calls for service occurring in December and January $(-57.13$ and -53.19 percent, respectively). The March/April peak is also present in each of the other incident types, though far less pronounced. The least monthly variation is found with the VF type followed closely by PFs. In addition to the March/April 


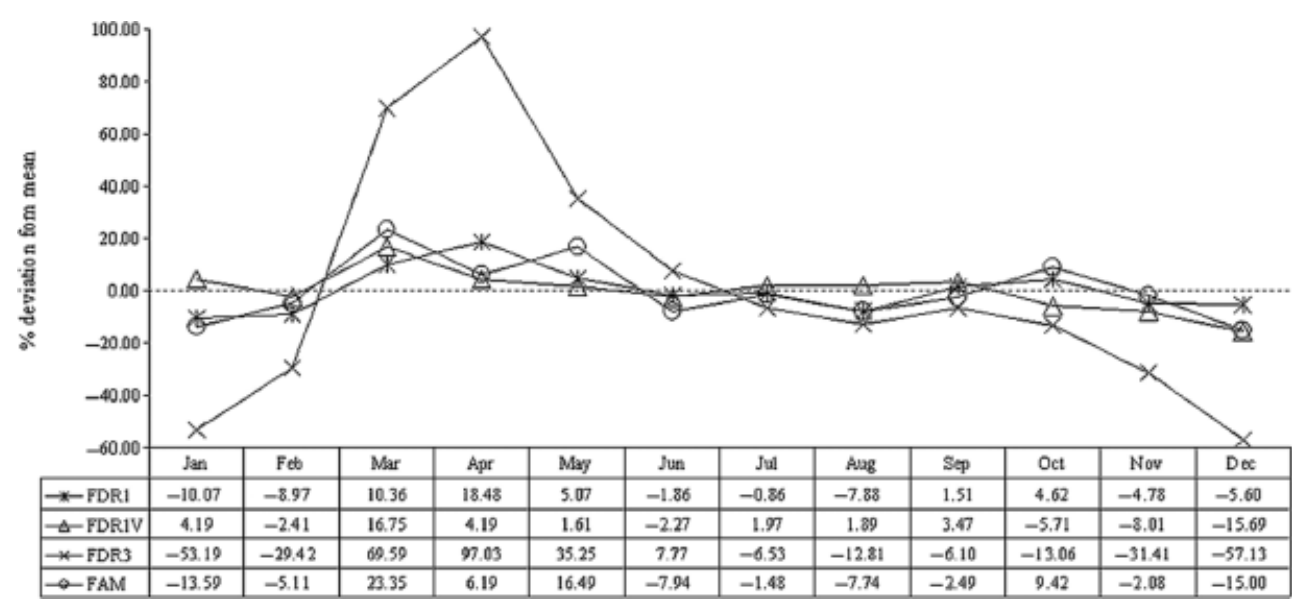

Figure 3 Monthly distribution.

peak, FA has a secondary peak in October $(9.42$ percent).

Daily Each incident type displays a similar daily pattern of greatest positive deviations during the weekend with fewer incidents than the mean occurring throughout the weekdays (Figure 4). The largest deviations from the mean are with VFs that exhibit the largest skew toward the weekend days. This is followed by SFs that is the only incident type to show a positive deviation for a weekday (Monday).

Hourly The greatest deviation from the mean hourly rate is displayed by SF with the peak times being between 19:00 and 20:30, falling below the mean between the hours of 22:30 and 13:30 (Figure 5). A similar distribution is

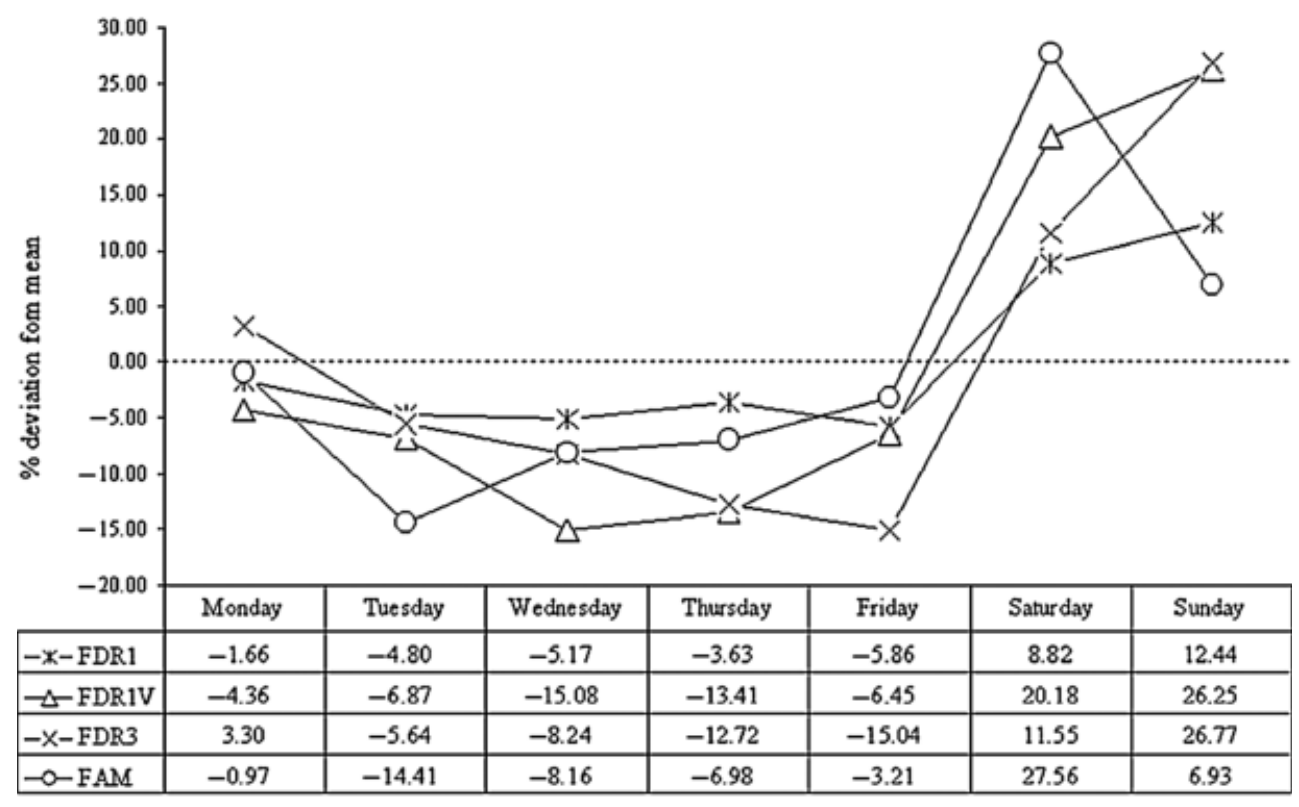

Figure 4 Daily distribution. 

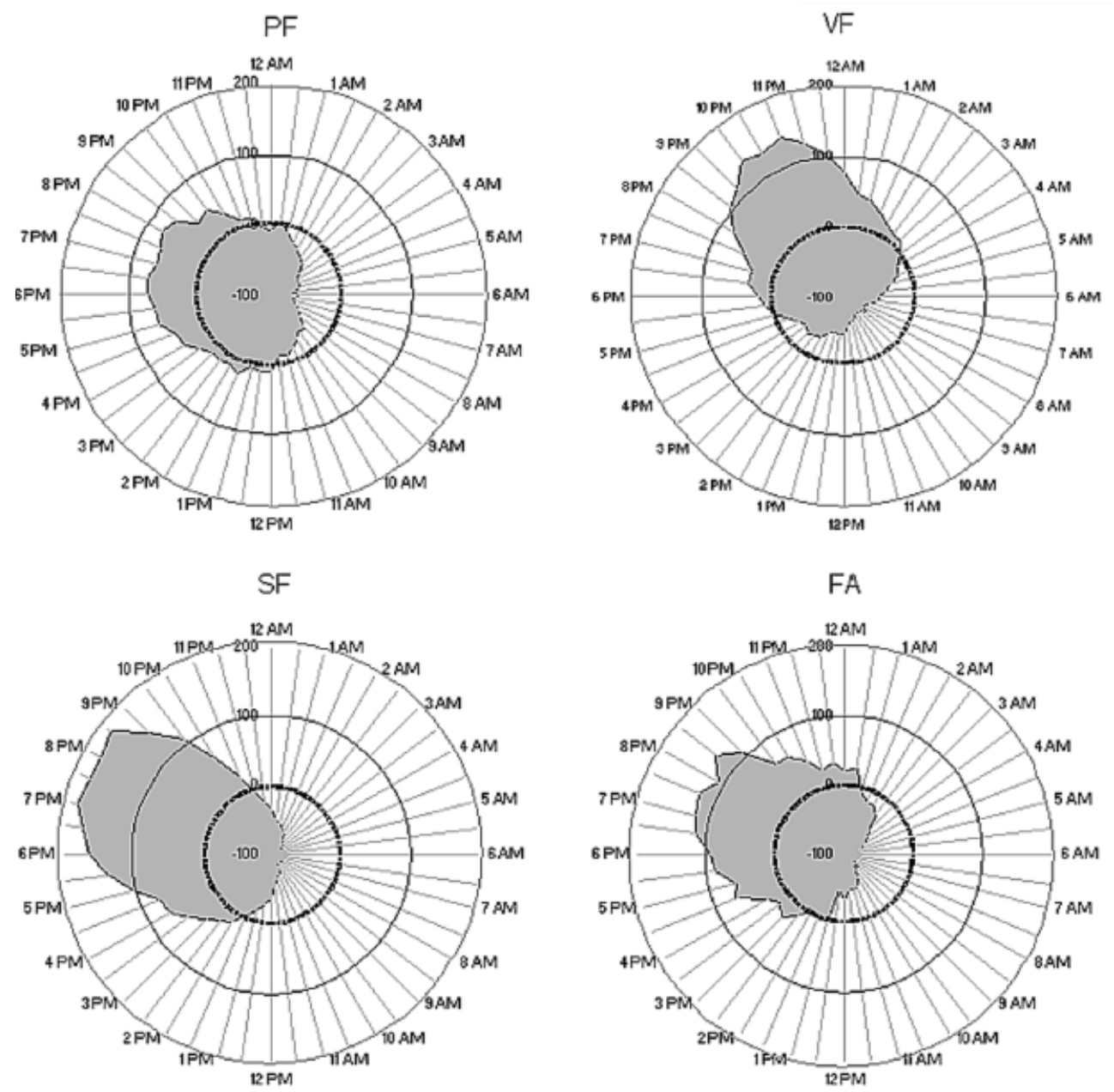

Figure 5 Hourly distribution. $P F=$ property fire; $V F=$ vehicle fire; $S F=$ secondary fire; $F A=$ false alarm.

also exhibited by FA, with a peak time of around 20:30, falling below the mean slightly later than the SF type (00:30 and 14:30). VF has the latest peak times (21:30-22:30) and only falls below the mean hourly rate from 03:30 until 17:00. PF shows the least deviation from the mean hourly rate in comparison to the other incident types, with peak times between 17:00 and 20:30, and falling below the mean from 23:30 and 12:00.

\section{Spatial-Temporal Dynamics}

The results presented thus far have highlighted that fire is not evenly spread in either space or time, and that this unevenness varies by incident type. However, the results presented are inca- pable of demonstrating the interaction between space and time. Using comaps it is possible to find out if the same bigh-risk areas are subject to temporal fluctuations in fire incidence, and to investigate whether temporal fluctuations are specific to areas that are not typically within bigh-risk locales. Finally, the effect of incident type is also investigated. Using both univariate and bivariate plots ( for each conditioning variable: month, day, and hour), spatiotemporal variations were investigated for each of the incident types, of which a subset is discussed in this article.

The univariate comap of FA conditioned by hour (Figure 6) shows a change in risk over the 
530 Volume 59, Number 4, November 2007
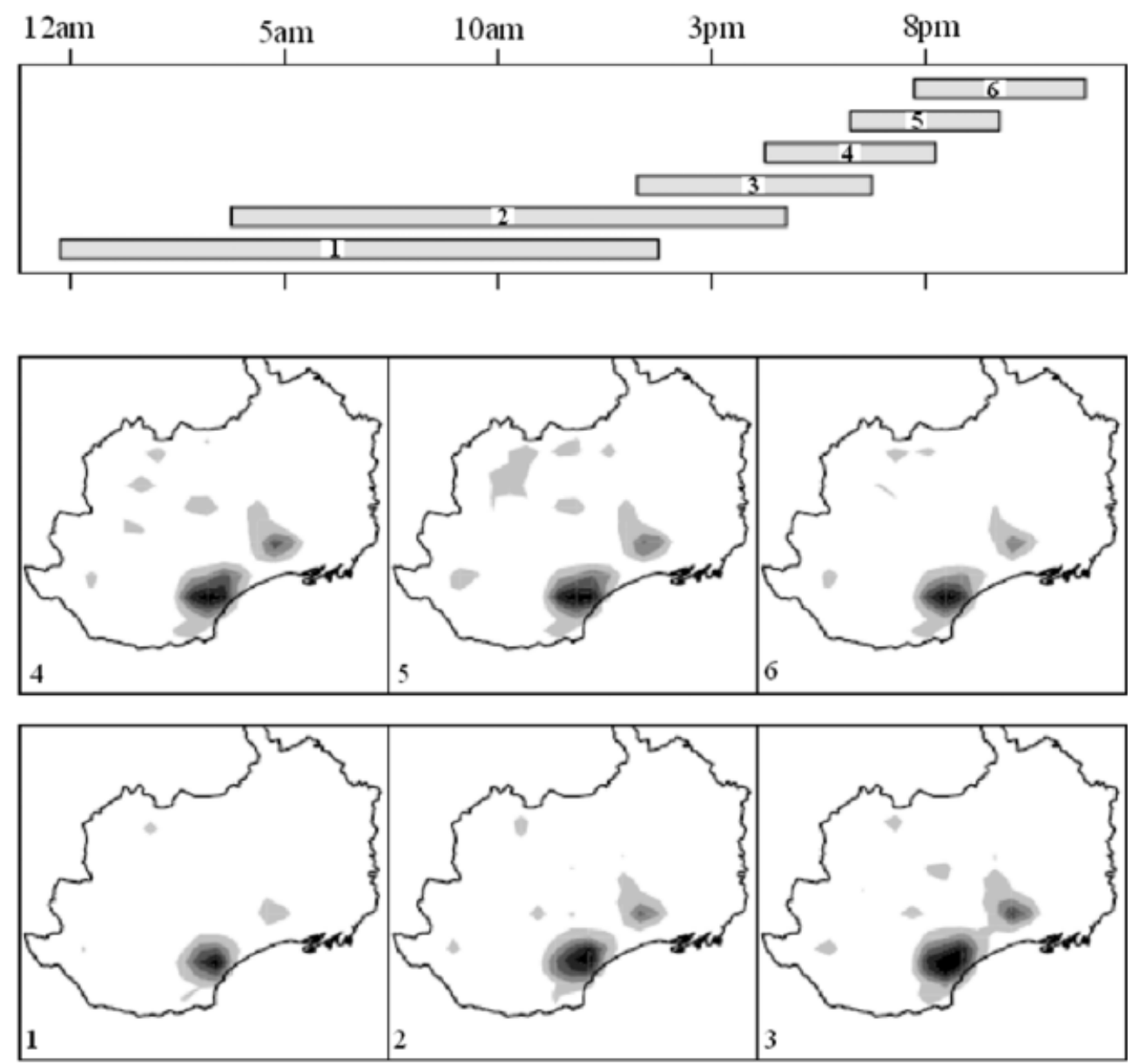

0

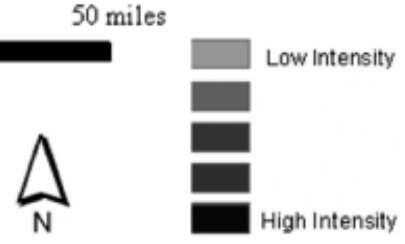

Figure 6 Univariate comap for false alarms (by the hour).

24-hour period. There is a relatively high concentration of FA incidents throughout the entire period in the Cardiff region, but other areas exhibit changes in their relative risk. The Newport region shows a low intensity of incidents during the early hours of the morning (see panel 1), then increasing intensity through the day until it forms a corridor connecting to the Cardiff region during the early afternoon and early evening (see panel 3). Other regions beyond those of Cardiff and Newport appear to be most prevalent in panel 5, representing incidents during the evening (from around 18:00 to around 22:00).

The univariate comap of PF conditioned by hour (Figure 7) shows very little change over the 24-hour period. The main concentration in the Cardiff region remains consistent throughout the period, as do the concentrations in the surrounding regions, suggesting that increases in 


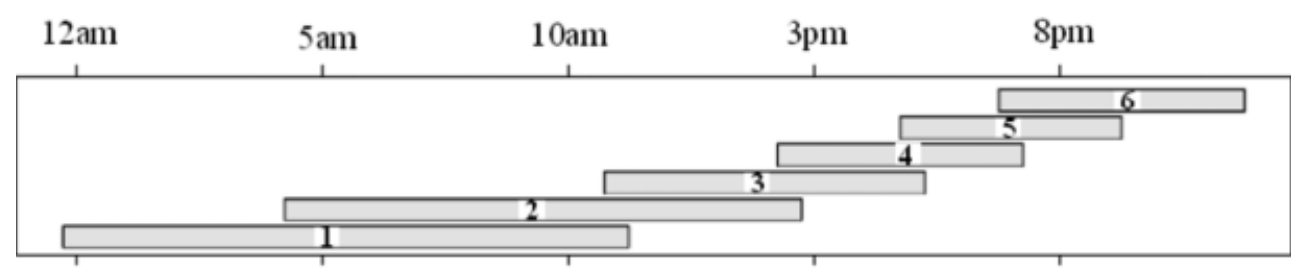
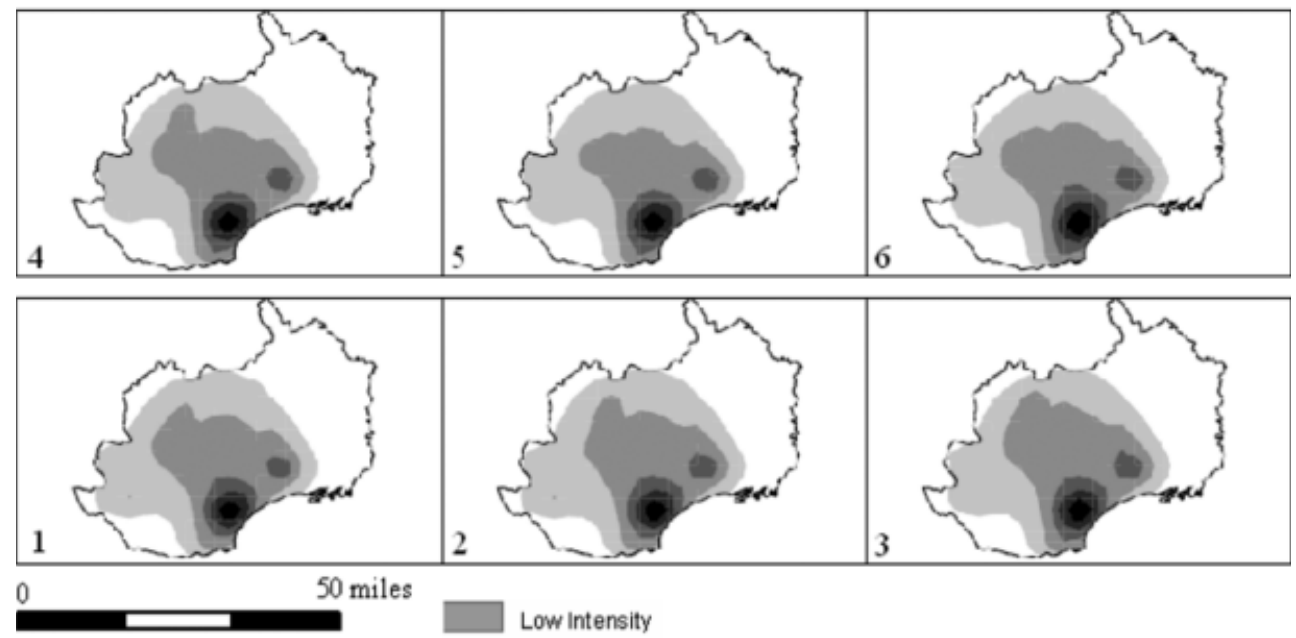

Low Intensity

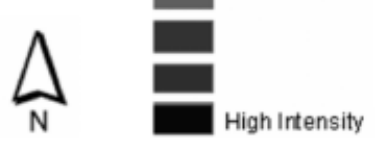

Figure 7 Univariate comap for primary fires (by the hour).

PFs are not spatially differentiable-simply the same areas are subject to the increases and decreases in household fires.

Figure 8 shows the bivariate comap of SF by hour and month. Here distinct spatial differences can be seen in both conditioning variables. First, over a 12-month period the location of the highest intensity varies dramatically over monthly intervals. The Cardiff region has the highest intensity between June and February, but between March and May the highest intensity shifts to the northeast of Pontypridd. Spatial differences are also evident by hour, with the greatest variation being in the October to February period where late afternoon/early evening shows the development of higher incident intensities around the Pontypridd region (see panel 2A).
Finally, the bivariate VF comap conditioned by hour and day of week (Figure 9) shows the main intensity areas (in and around the Cardiff region) to be stable throughout the day and week. The main variations are by hour (particularly between Mondays to Thursdays) where the Pontypridd region experiences a rise in intensity during the afternoon through to the evening/night that dissipates during the early hours of the morning through to the afternoon. This phenomenon is less pronounced on Fridays, Saturdays, and Sundays where the hourly variation exhibits less of an influence.

\section{Discussion}

A primary aim of this study was to use visualization techniques based on comaps and $\mathrm{KDE}$ 

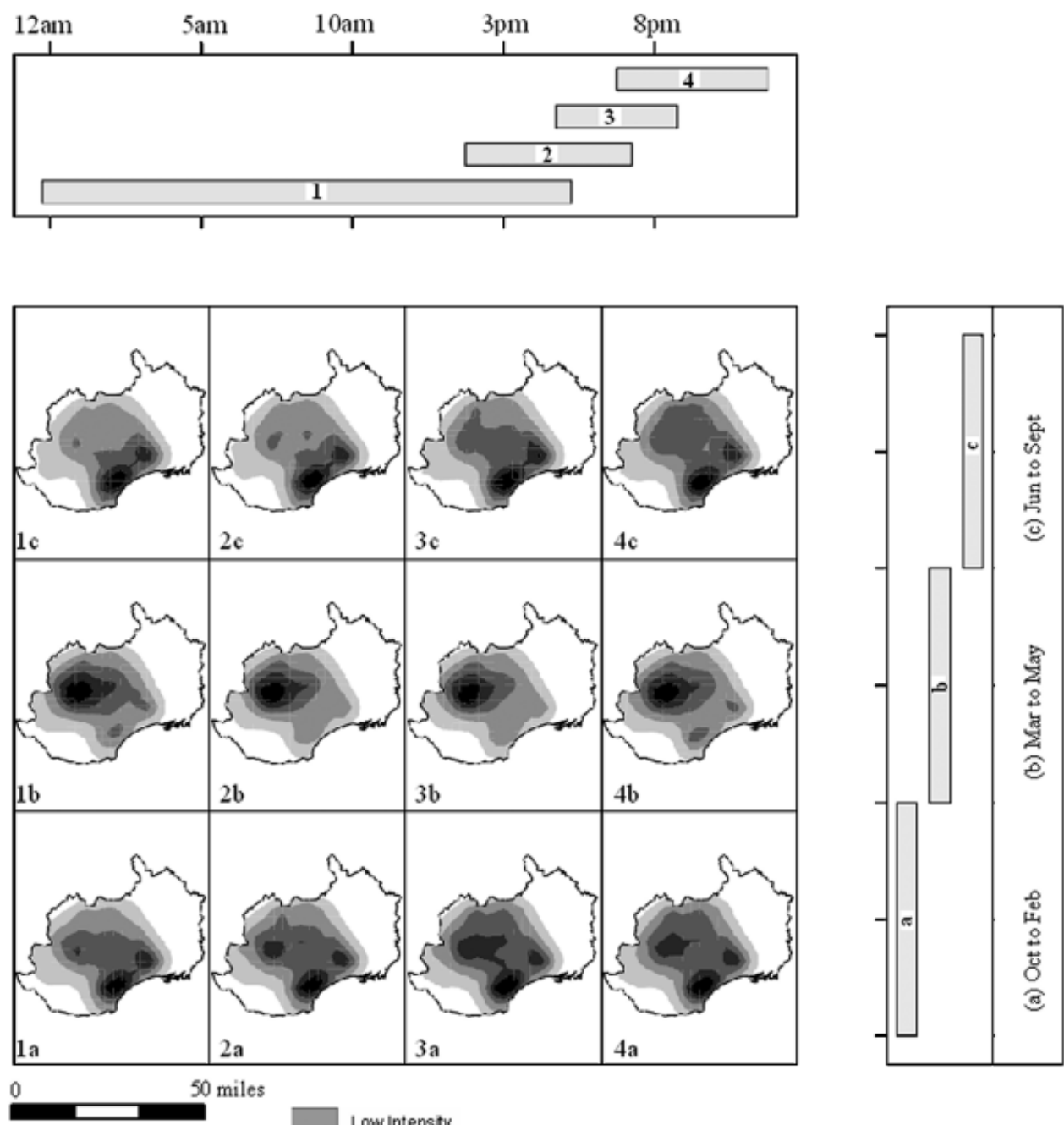

Low Intensity
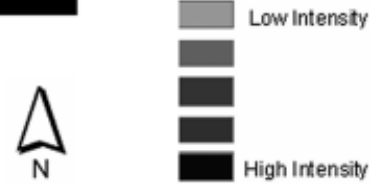

Figure 8 Bivariate comap for secondary fires (by the hour and month).

to investigate spatial patterns of fire incidents and their variation over time. There has been some use of such techniques in investigating spatiotemporal patterns in crime incidence (see, e.g., Brunsdon, Corcoran, and Higgs 2007), but there has been less research focusing on variations in fire incidence. We redress this gap in the research through the use of a range of spatial, temporal, and spatiotemporal techniques ap- plied to disaggregate fire incident data. To date, $\mathrm{KDE}$ has been applied in fields such as crime and health studies, but its application to fire data has yet to be fully explored. In addition, whereas the majority of previous studies utilized relatively coarse spatial and temporal unit of analysis, the motivation of this study was to explore microscale spatial and temporal dynamics using the disaggregated data that are increasingly be- 

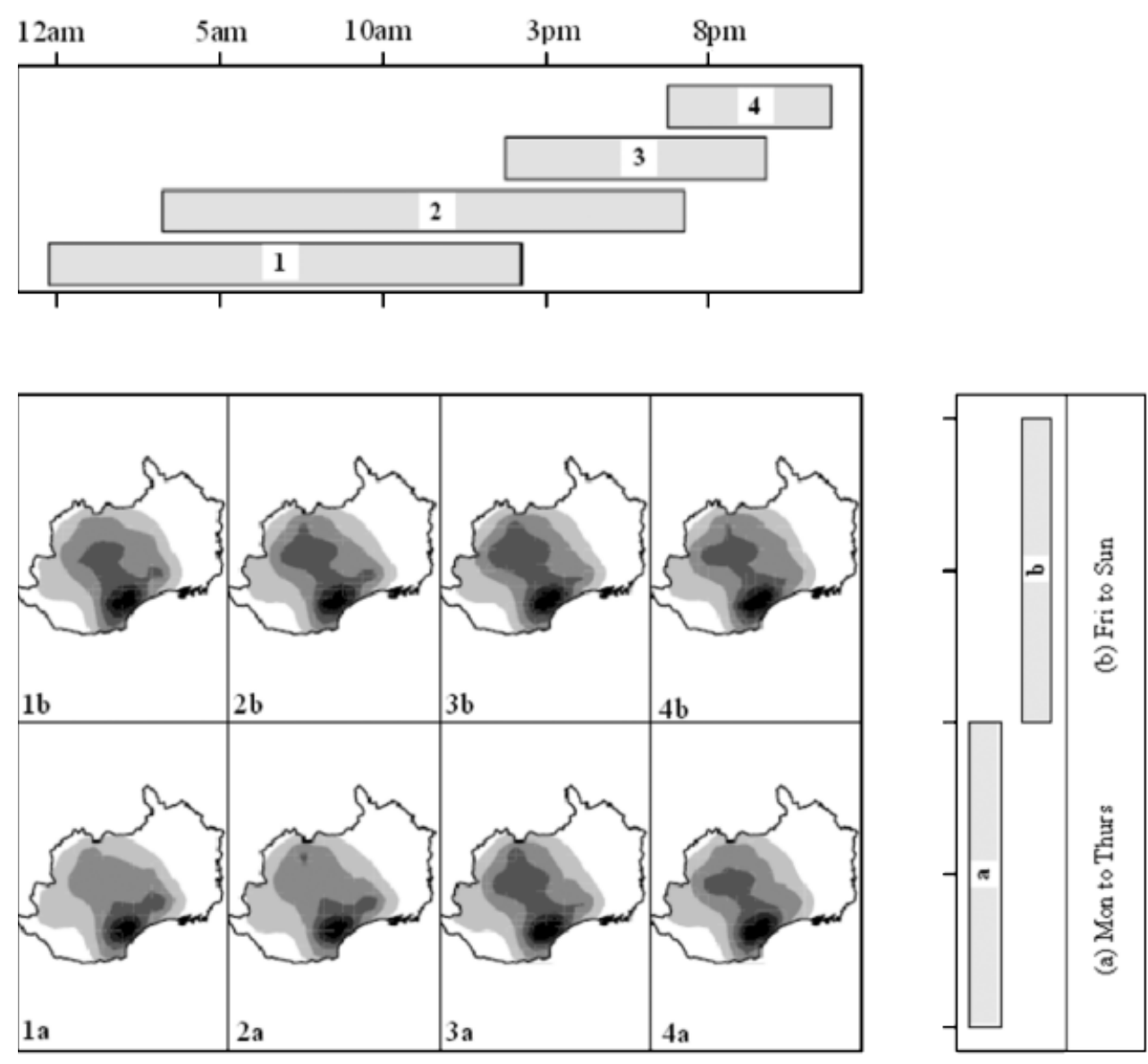

0

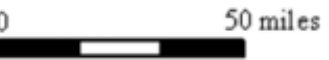

Low Intensity
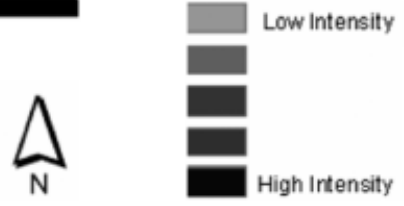

Figure 9 Bivariate comap for vehicle fires (by the hour and day of week).

ing collected by emergency services. Here micro is defined as point data for each fire incident (each fire incident having an $x, y$ coordinate) containing an hour and date pertaining to the timing of the call for service.

Our findings suggest that fire incidents vary in both time and space, with the degree of variation being dependent on incident type. The types of techniques developed in our research provides a means through which we can investigate such trends at detailed spatial scales as well as examine potential reasons for such trends in any future research. This could involve a detailed study of the socioeconomic circumstances of each of the areas and a detailed consideration of any temporal variations following fire prevention strategies (e.g., the installation of smoke detectors). Of the four incident types investigated, PFs varied the least over both space and time, whereas VFs, SFs, and FAs all showed larger temporal deviations, with the trend being toward the weekend and 
evenings/nighttime. SFs showed the largest monthly variation with significant increases during March, April, and May that additionally showed the most distinct spatial deviation from the spatial patterns exhibited in other months. Differences in spatial pattern were also evident in VFs and FAs, the tendency being for the Cardiff region to consistently contain the majority of the incidents (particularly the case for FAs), the variation being in outlying regions, largely throughout the week and over a 24-hour period. To examine any differences between urban and rural/suburban patterns, further research (utilizing additional contextual data such as the census) is needed to explain these patterns in more detail and to see if they are related to, for example, levels of urbanization within the study area. The availability of a small area classification of census areas in the United Kingdom by the Office of National Statistics for the 2001 Census of Population could be useful in this regard.

To date, the majority of studies on trends in fire incidence have generally been conducted at relatively coarse spatial scales (such as citywide and regional levels) for which census data are available. The lack of precision in geocoding in the past has also meant that only aggregate analysis was possible. However, the availability of detailed disaggregate data sources of the type we have had access to in this study and the increasing use of GPSs within the emergency services have led to the development of rich sources of data that can be used within GIS to investigate such trends. Jennings (1998) suggests that there has been a lack of microlevel fire studies to date and that GIS can be used to examine relationships between fire incidence and the built environment. Many of the techniques we have been developing during the course of our research are not currently available in commercial GIS packages. Previous studies have also traditionally used relatively coarse temporal units such as monthly and annual counts of fire ncidence. Some notable exceptions to this are evident in the work of Dodge (1996), who identified that the weekly distribution of fire incidents is not uneven and that small fires and FAs tend to occur on Fridays and Saturdays, whereas nonmalicious false alarms (deemed as good intent) are more prevalent during weekdays. A comparable finding is reported in our study whereby the FA incident type exhibits similar behavior at the daily level of analysis.

Our future work will involve a more in-depth investigation of the findings presented here. Such research could follow a number of strands. First, given a more detailed breakdown of VFs into deliberate or nondeliberate types (with suitable confidentiality/privacy constraints), more detailed conclusions could be drawn. In addition, a more comprehensive analysis of $\mathrm{PF}$ incidents could be obtained with the use of further contextual data such as type of house, tenancy, gender, and presence of fire alarm equipment. Such a level of analysis is supported by the work of Lapidus et al. $(1998,105)$, for example, who found in their study of residential fire incidence in Hartford, Connecticut (for 1992-1994), that a large proportion of homes in census tracts with high levels of house fires either had no smoke detectors present or had smoke detectors that were nonfunctional. Other potential factors could be used to explore temporal distributions such as temperature and other climatic variables in relation to forest or heathland fires and the spatial relationship between teenage population and, for example, hoax calls and arson fires. Dependent on the availability of appropriate data sources for our study area, this analysis could be expanded to try to explain the findings presented in this article. Finally, further work is now required to assess the transferability of the findings presented here to other contexts and international settings.

\section{Conclusions}

The majority of studies to date in the area of fire applications have used GIS to examine aggregate incident patterns in relation to socioeconomic data (largely at the level of census tracts). These have focused primarily on spatial snapshots of fire incidence in relation to, for example, small area data using traditional correlation and regression techniques. The application of mapping has proved to be useful for front-line fire services and community safety teams in targeting prevention initiatives such as educational programs and digital mapping, with the result that GIS software has been increasingly taken up by emergency services in fire planning, fire safety, and risk-assessment applications. Relatively few studies have investigated the spatiotemporal dynamics for different types 
of fire incidents and the potential influence of spatial and temporal variations in socioeconomic conditions. The availability of disaggregate sources of data related to incidents provides the potential for a more detailed analysis of such patterns. To show how fire incidents vary in both time and space, this study utilized visualization techniques previously applied to crime and health data. Our findings suggest that such trends differ according to incident type and have the potential to provide important exploratory analysis prior to more detailed investigations. These investigations could involve the use of contextual data (e.g., land use, type of residence, and construction type) in addition to more detailed descriptions of incident types (such as the presence of smoke alarms, extent of fire and smoke damage, response times, and the number of recorded casualties and fatalities) in attempting to explain such patterns. More generally, the application of these techniques has the potential to inform policy decisions both from a reactive, resource-allocation perspective and from a more proactive, preventative perspective such as spatial targeting of preventative measures.

\section{Literature Cited}

ArcGIS. 2005. Version 9.1. Redlands, CA: Environmental Science Research Institute.

Australasian Fire Authorities Council. 2005. Accidental fire fatalities in residential structures: Who's at risk? Available at http://www.afac.com.au/awsv2/ publications/documents/ResidentialFireFatalities ReportOctober2005.pdf (last accessed 19 February 2007).

BBC News. 2006. $£ 700,000$ cost of hoax calls, 16 February 2006. Available at http://news.bbc.co.uk/ 2/hi/uk_news/wales/4715834.stm (last accessed 19 February 2007).

Boba, R. 2005. Crime analysis and crime mapping. Beverly Hills, CA: Sage.

Brunsdon, C. 2001. The comap: Exploring spatial pattern via conditional distributions. Computers, Environment and Urban Systems 25:53-68.

Brunsdon, C., J. Corcoran, and G. Higgs. 2007. Visualising space and time in crime patterns: A comparison of methods. Computers, Environment and Urban Systems 31:52-75.

Chainey, S., and J. H. Ratcliffe. 2005. GIS and crime mapping. London: Wiley.

Chandler, S. E., A. Chapman, and S. J. Hallington. 1984. Fire incidence, housing and social conditions: The urban situation in Britain. Fire Prevention 172:15-20.
Cleveland, W. S. 1993. Visualizing data. Summit, NJ: Summit.

Corcoran, J., G. Higgs, C. Brunsdon, A. Ware, and P. Norman. 2007. The use of spatial analytical techniques to explore patterns of fire incidence: A South Wales case study. Computers, Environments and Urban Systems. Available at ScienceeDirect http://www. sciencedirect.com/(last accessed 6 July 2007).

DiGuiseppi, C., P. Edwards, C. Godward, I. Roberts, and A. Wade. 2000. Urban residential fire and flame injuries: A population based study. Injury Prevention 6:250-54.

Dodge, M. 1996. The visualisation and analysis of fire incidents using GIS. In Proceedings of the second joint European Conference \& Exhibition on Geographical information (Vol. 1): From research to application through cooperation, ed. M. Rumor, R. McMillan, and H. F. L. Ottens, 592-601. Amsterdam: IOS.

Eck, J., S. P. Chainey, J. Cameron, M. Leitner, and R. Wilson. 2005. Mapping crime: Understanding botspots. Washington, DC: National Institute of Justice. Available at http://www.ncjrs.gov/ pdffiles1/nij/209393.pdf (last accessed 19 February 2007).

Goldsmith, V., P. G. McGuire, J. B. Mollenkopf, and T. A. Ross. 2000. Analyzing crime patterns: Frontiers of practice. Thousand Oaks, CA: Sage.

Harries, K. 1999. Mapping crime: Principle and practice. Washington, DC: National Institute of Justice.

Hernandez-Leal, P. A., M. Arbelo, and A. GonzalezCalvo. 2006. Fire risk assessment using satellite data. Advances in Space Research 37 (4): 741-46.

Hirschfield, A., and K. Bowers. 2001. Mapping and analysing crime data: Lessons from research and practice. London: Taylor \& Francis.

Jaiswal, R. K., S. Mukherjee, K. D. Raju, and R. Saxena. 2002. Forest fire risk zone mapping from satellite imagery and GIS. International fournal of Applied Earth Observation and Geoinformation 4: $1-10$.

Jennings, C. R. 1998. Urban fire risk: Using GIS to connect fire, census and assessor's data. Regional Science Review 17:105-12.

- 1999. Socioeconomic characteristics and their relationship to fire incidence: A review of the literature. Fire Technology 35(1):7-34.

Lapidus, G., S. McGee, R. Zavoski, E. Cromley, and L. Banco. 1998. Using a geographic information system to guide a community-based smoke detector campaign. In Geographic information systems in public health: Proceedings of the $3 r d$ National Conference, ATSDR, Agency for Toxic Substances and Disease Registry, ed. R. C. Williams, M. M. Howie, C. V. Lee, and W. D. Henriques, 103-8, 18-20 August, Atlanta, GA. Available at http://www.atsdr.cdc.gov/gis/confer ence98/proceedings/pdf/lapidus.pdf (last accessed 9 July 2007). 
Leipnik, M. R., and D. P. Albert. 2003. GIS in law enforcement: Implementation issues and case studies. London: Taylor \& Francis.

Liu, N., B. Huang, and M. Chandramouli. 2006. Optimal siting of fire stations using GIS and ANT algorithm. Fournal of Computing in Civil Engineering (ASCE) 20 (5): 361-69.

Merrall, S. 2001. GIS for spatial analysis of fire incidence: Identification of social, economic and environmental risk indicators. In Mapping and analysing crime data: Lessons from research and practice, ed. A. Hirschfield and K. Bowers, 156-83. London: Taylor \& Francis.

ODPM. 2005a. Fire statistics monitor. Office of the Deputy Prime Minister Available at http:// www.communities.gov.uk/pub/958/FireStatistics MonitorQ2andQ32004PrintversionPDF174Kb_ id1124958.pdf (last accessed 19 February 2007).

- 2005b. Integrated risk management planning: Guidance notes. Office of the Deputy Prime Minister. Available at http://www.communities.gov.uk/ index.asp? is=1124262 (last accessed 19 February 2007).

Ormsby, D. 2005. Fighting and preventing fires with geographic intelligence. Geoconnexion September/ October: 34-35.

Paulsen, J., D. Robinson, and M. B. Robinson. 2004. Spatial aspects of crime: Theory and practice. Boston: Pearson Education.

Pew, K. L., and C. P. S. Larsen. 2001. GIS analysis of spatial and temporal patterns of human-caused wildfires in the temperate rainforest of Vancouver Island, Canada. Forest Ecology and Management 140:1-18.

Silverman, B. W. 1986. Density estimation for statistics and data analysis. London: Chapman and Hall.

Tufte, E. R. 1990. Envisioning information. Cheshire, CT: Graphics Press.

Vakalis, D., H. Sarimveis, C. T. Kiranoudis, A. Alexandridis, and G. Bafas. 2004a. A GIS based operational system for wildland fire crisis management. I, Mathematical modelling and simulation. Applied Mathematical Modelling 28:389-410. 2004b. A GIS based operational system for wildland fire crisis management. II, System architecture and case studies. Applied Mathematical Modelling 28:411-25.

Weisburd, D., and J. T. McEwen. 1997. Crime mapping and crime prevention. Monsey, NY: Criminal Justice Press.

JONATHAN CORCORAN is a Research Fellow in the University of Queensland Social Research Centre (UQSRC) at the University of Queensland, Brisbane, Queensland, Australia 4072. E-mail: jj.corcoran@ uq.edu.au. His research interests include the application of geographical techniques for urban modeling and the use of geoanalytical, geo-visualization, and prediction techniques primarily applied to emergency service data.

GARY HIGGS is a Professor in the GIS Research Centre, Faculty of Advanced Technology, University of Glamorgan, Pontypridd, United Kingdom CF37 1DL. E-mail: ghiggs@glam.ac.uk. His research interests include spatial analysis, GIScience, and medical geography.

CHRIS BRUNSDON is a Professor in the Department of Geography, University of Leicester, University Road, Leicester, United Kingdom LE1 7RH. E-mail: cb179@leicester.ac.uk. His has worked for a number of years in the area of spatial analysis and spatial statistics, both in his current position and at the Universities of Glamorgan and Newcastle upon Tyne.

ANDREW WARE is a Professor in the Faculty of Advanced Technology, University of Glamorgan, Pontypridd, United Kingdom CF37 1DL. E-mail: jaware@glam.ac.uk. His main research interest is in the deployment of intelligent computer systems to help solve realworld problems. 